Journal of the

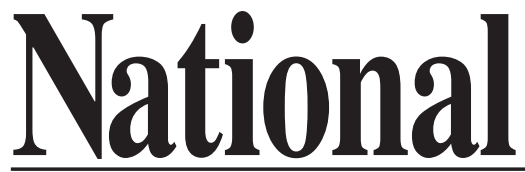

Academy or

Forensic
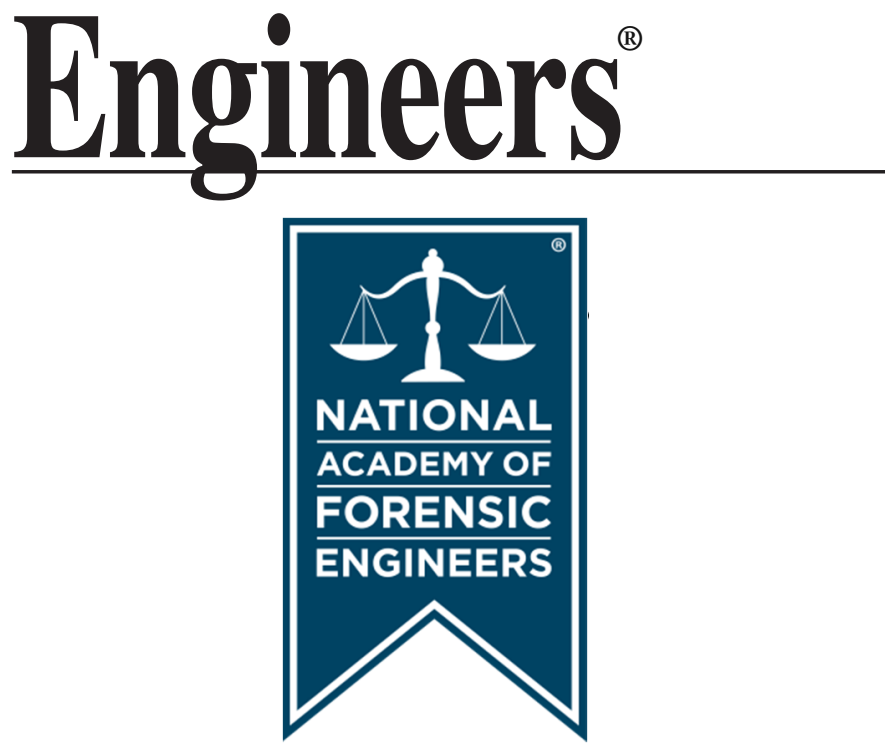

http://www.nafe.org

ISSN: 2379-3252

Vol. XXIII No. 2 December 2006 


\title{
Forensic Engineering Analysis of Pedestrian Trauma Using BioMedical and Accident Reconstruction Engineering Methods
}

by Laura Liptai, Ph.D. (NAFE C339)

Rowland Lamb, P.E. (NAFE 690S)

\begin{abstract}
This study examines the theory and methods of forensic engineering analysis applied to pedestrian trauma and fatality. Optimally approached with the cols lective analysis of both the Accident Reconstruction Engineer and BioMedical Engineer, the Accident Reconstruction Engineer translates the physical evidence related to the vehicle and scene while the BioMedical Engineer interprets the physical evidence related to the human body in the biological tissues. With both disciplines, a collaborative understanding can be gained using the vehicular and human tissue physical evidence available.
\end{abstract}

The case presented involves a pedestrian verses an automobile. The collision between a pedestrian and automobile resulted in the death of the pedestrian. Physical evidence on the vehicle included: a dent in the front right fender; a spider web glass fracture pattern on the windshield in the lower right corner; and a dent in the right A-pillar.

This study will also demonstrate what can be learned from the absence of classic physical evidence on the vehicle and on the pedestrian as well as the determination of when a kinematic study is appropriate for pedestrian verses automobile investigation.

\section{Introduction}

Collisions between pedestrians and automobiles are a leading cause of fatalities. This work seeks to integrate Accident Reconstruction Engineering with BioMedical Engineering in an attempt to determine the sources of the physical evidence and who was at fault in the incident. 
Facts:

- A female college co-ed was jogging while wearing headphones when she came to an intersection with a marked crosswalk.

- An SUV was stopped for a red light in the curb lane when the pedestrian first arrived at the intersection.

- An older model compact sedan was traveling in the center lane adjacent to the SUV. See Figures $1 \mathrm{~A}$ and $1 \mathrm{~B}$.

- The speed limit was thirty (30) miles per hour.

- The pedestrian signal functions on command, so the "walk" indicator will only activate when a pedestrian pushes the button. The walk indicator was not activated by the pedestrian in this incident.

- After allegedly being waved across by the occupants of the SUV in the curb lane, the pedestrian jogged past the front of the SUV and a collision occured between the compact sedan and the pedestrian.

- The collision resulted in the death of the pedestrian.

- Physical evidence on the vehicle included: a dent in the front right fender; a spider web fracture of the windshield in the lower right hand corner; and a dent in the right A-pillar approximately eighteen (18) inches up from the base.

- The pedestrian came to rest approximately thirty-two (32) feet past of the point of impact.

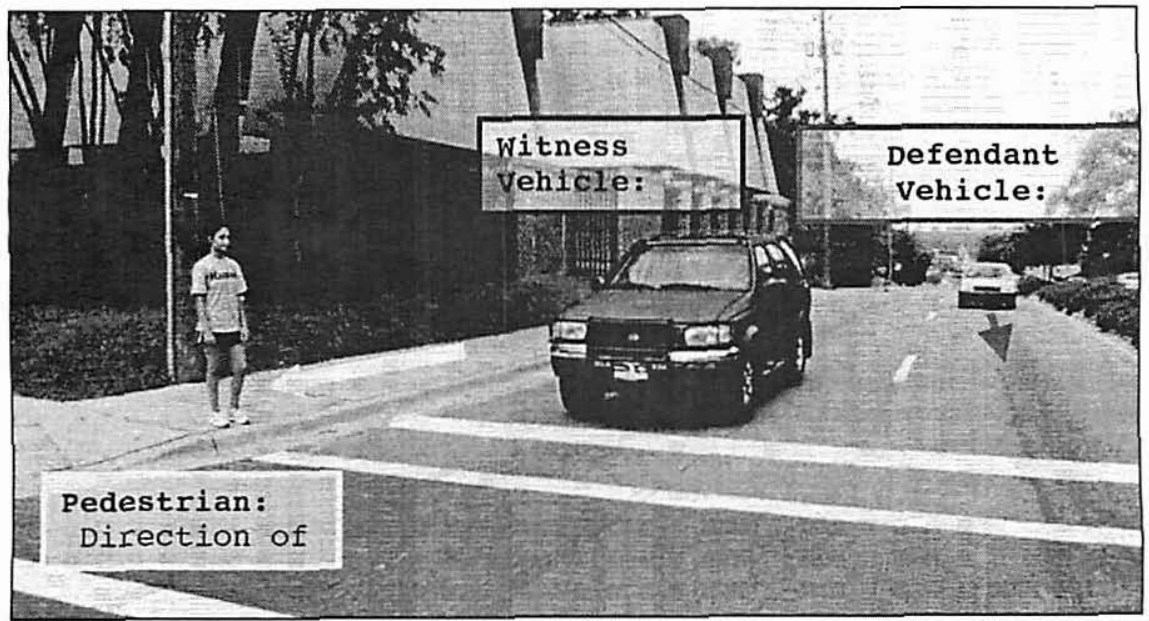

Figure 1A

Vehicle and Pedestrian Initial Juxtaposition:

Pedestrian Crossing in Front of Stopped SUV and Defendant Vehicle On-Coming 


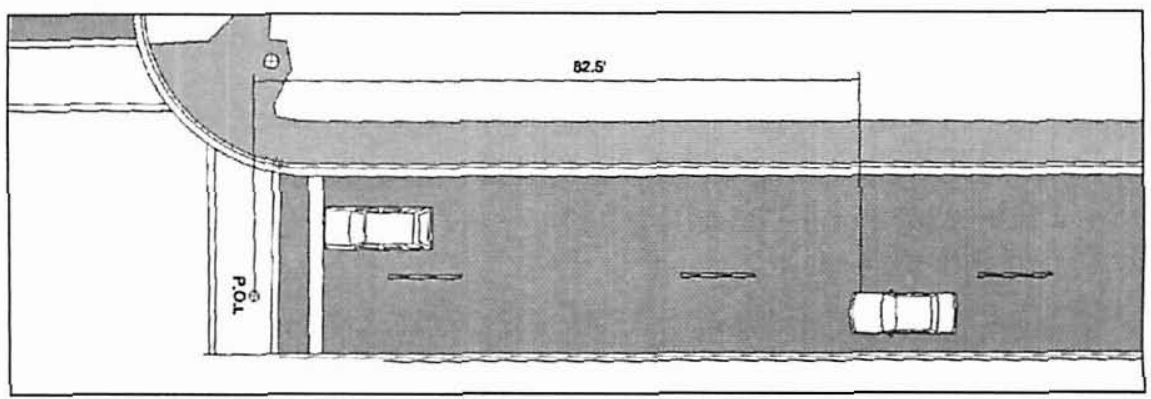

\section{Figure 1B}

Locations of the Two Vehicles and the Pedestrian at the

"Point of No Return" for the Defendant

In Contention:

- Who was at fault for the collision: the pedestrian, the driver of the sedan or the occupants of the SUV who allegedly waved the pedestrian across?

- Was the pedestrian signal in the "Walk," "Caution" or "Don't Walk" phase?

- Was the signal red or green for the southbound traffic when the pedestrian left the curb?

- Did the pedestrian run into the side of the vehicle or did the vehicle hit the pedestrian?

- Could the driver of the sedan have been able to perceive and react to avoid the collision?

\section{Analysis}

Initially the accident reconstruction engineer was engaged by defense counsel to reconstruct the events of the crash. During the analysis, the engineer recommended a BioMedical Engineering expert to interpret the physical evidence on the pedestrian.

The Biomedical Engineer and the Accident Reconstruction Engineer worked collaboratively to evaluate the combination of the information gained from the vehicular and human tissue physical evidence.

The Biomedical Engineer was responsible for the:

- interpretation of the physical evidence within the biological tissues;

- calculation of the acceleration and speed of the pedestrian;

- kinematics of the impact of the pedestrian's body with the automobile; and 
- visibility of the vehicle to the pedestrian prior to leaving the sidewalk.

The Accident Reconstruction Engineer was responsible for the:

- determination of the speed of the vehicle;

- determination of the driver's ability to perceive, react and avoid the collision with the pedestrian; and

- determination of the traffic signal direction before and during the collision.

\section{Accident Reconstruction Engineering Analysis of Vehicle Speed}

The Accident Reconstruction Engineer conducted an analysis of the vehicle's speed (V) based on perception reaction plus braking using the following information provided by the investigating police officers.

(a) Estimated point of impact to the location where the car stopped was one hundred forty (140) feet.

(b) There were no skid marks.

(c) Driver stated that he "heard a thud... and thought someone had thrown a brick and hit his car" so he braked and pulled over.

From this information it was determined that:

- the perception reaction time (PRT) began at the point of impact, and

- the driver did not panic brake but did apply firm braking.

Total Distance $=$ Distance traveled during PRT + Distance traveled during braking; therefore,

$140^{\prime}=(P R T)(V)+\left(V^{2}\right) /((2)(32.2)(f))$ where " $\mathrm{f}$ " is defined as the coefficient of friction.

The driver testified that he initially "heard a thud" and subsequently observed the shattered wind shield at which time he braked and pulled over. Therefore, his perception begins at the point of impact and is drawn out as he looks to determine what happened. Utilizing audio only PRT's of one point five (1.5), two point zero (2.0), and two point five (2.5) seconds and drag factors of the vehicle braking from zero point four $(0.4)$ to zero point six $(0.6)$, the Accident Reconstruction Engineer arrived at calculated speeds of the defendant's vehicle of twenty three (23) to twenty nine (29) miles per hour.

Initially it was thought that the vehicle hit the pedestrian; however, the Biomedical Engineering analysis of the physical evidence determined that the 
pedestrian ran into the side of the sedan. This determination contributed to the Accident Reconstruction in determining the speed of the defendant's vehicle. Combining the knowledge that the pedestrian impacted the side of the vehicle with the understanding as to how the pedestrian ramps up the side of the vehicle, the Accident Reconstructionist was able to complete the analysis of the pedestrian carry.

To check the speed ranges, the Accident Reconstruction Engineer utilized the scene measurements for the pedestrian body point of rest and the information obtained from the BioMedical Engineering expert, including the contact along the surface of the vehicle and trauma physical evidence, to determine pedestrian carry distance.

Since the pedestrian was never completely mounted on the car, the engineer used a pedestrian speed $(\mathrm{Vp})$ of seventy $(70)$ percent of the vehicle speed $(\mathrm{V})$ immediately following impact. The pedestrian traversed an estimated five (5) feet along the vehicle before dropping off the vehicle, impacting the pavement and sliding to a stop at the point of rest.

Therefore if,

$$
\begin{aligned}
& V_{p}=0.7 \mathrm{~V}, \\
& D_{\text {total }}=D_{\text {ped } / \text { car }}+D_{\text {ped/air }}+D_{\text {ped } / \text { street }}=32 \text { feet, }
\end{aligned}
$$

Based on research, the drag factor for the pedestrian along the vehicle ranged from zero point three $(0.3)$ to zero point seven $(0.7)$. Using a static slipometer, clothing materials comparable to those worn by the pedestrian were tested on the exemplar car. An average drag coefficient of zero point four $(0.4)$ was determined using this method.

Based on research, the drag factor for the "tumble" and "skid" of the pedestrian on the street was determined to be approximately zero point six (0.6).

Solving for the initial velocity, utilizing linear momentum, it was determined that the velocity of the compact sedan at the point of impact was approximately twenty-five (25) miles per hour. This assumed a constant velocity of the vehicle while the pedestrian was in contact, assuming the perception/reaction of the driver did not begin until the point of impact.

\section{Signal Color Discussion}

Examination of different and commonly contradicting witness statements can be an important issue for forensic engineers. In this case, one witness traveling in the opposite direction clearly remembers the signal being green since 
she crossed an intersection one block to the south. She further states that her speed was twenty (20) miles per hour as she traveled the one block. This would mean that the signal had been green for at least eleven point six (11.6) seconds.

The two witnesses in the SUV both stated that the light was red when the pedestrian left the sidewalk. After the collision, they looked up and the light was green. This could mean that the driver of the sedan either ran the red light or the light turned green right as the sedan got to it.

Was the light really green for over eleven (11) seconds or was it red when the collision occurs? Were the witnesses in the SUV more focused on the jogger and the three (3) children in the back seat so the change from red light to green light went unnoticed? In this case, it was undisputed that she crossed on a "Don't Walk" pedestrian signal, and the color of the signal for the defendant was unknown.

\section{Biomedical Engineering Analysis}

The BioMedical Engineering analysis began with a review of the vehicle photos, scene photos, medical records, autopsy photographs, and the pedestrian's clothing. From this, every trauma on the pedestrian's body was documented in description as well as photographically. Even the evidence on the pedestrian's watch provided insight into the trajectory. The physical evidence on the defendant's vehicle is pictured as Figure 2. The decedent's primary trauma map diagram is shown as Figure 3.

\section{Other experts opined that the vehicle had run into the pedestrian while the pedestrian was attempting to turn around in the crosswalk.}

Using a displaced front right (passenger side) headlight as evidence, other experts opined that the vehicle had run into the pedestrian. This theory was

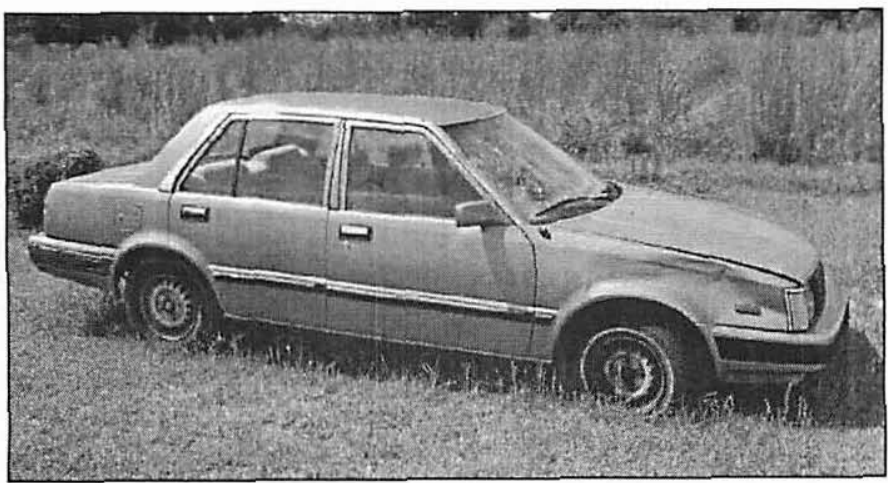

Figure 2

Defendant Vehicle Damage -

Note passenger side front quarter panel damage 


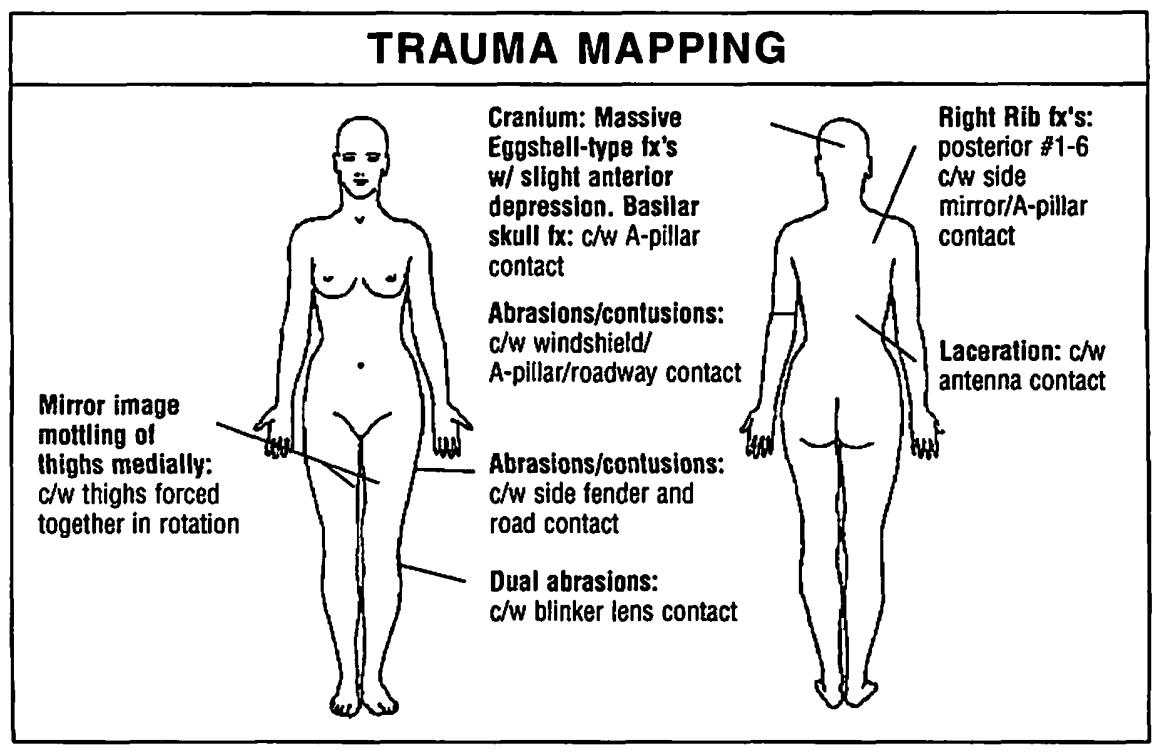

Figure 3

Trauma Map of the Primary Injuries Sustained by the Pedestrian

based on the observation that most of the trauma on the posterior aspect of the decedent's body, occurred while the pedestrian was attempting to turn around part way across the street to escape impact with the defendant's vehicle. The medical examiner opined that the "mirror image type contusion-like mottling of the opposing surfaces on the right and left thighs medially" traced to the pedestrian impacting the front of the defendant's vehicle.

The BioMedical Engineer determined through investigation that:

- the displaced headlight was from a previous unrelated incident;

- the absence of hood damage was not consistent with a classic frontal pedestrian impact;

- the pedestrian sustained no bumper related trauma;

- the "mirror image type contusion-like mottling of the opposing surfaces on the right and left thighs medially" traced to the medial thighs being rubbed against each other forcefully in shear, when the pedestrian laterally impacted the passenger side quarter panel; and

- the pedestrian was not visible to the approaching vehicle at one point five (1.5) seconds before impact. 


\section{Could the pedestrian have dented the right front quarter panel with a glancing blow?}

An additional area of analytical exploration was the determination of whether a running one hundred twenty five (125) pound woman could have dented the right front quarter panel. The two approaches considered were experimental and theoretical. It was determined that it would be possible to develop a test that would generate a substantially similar pedestrian impact; however, the theoretical approach was used as a first order approximation.

To analyze whether the pedestrian's body could have deformed the right front quarter panel, the area of impact was modeled as a simply supported rectangular plate. All variables are defined in Figure 4 except "D" which equals the bending rigidity of the plate as a function of the Young's modulus (E), the poisson's ratio $(\mu)$ and the thickness of the plate $(t) . D=\left(E^{*} t^{3}\right) /\left[12\left(1-\mu^{2}\right)\right]$ This was appropriate as there were three (3) inches of structural clearance under the indented fender.

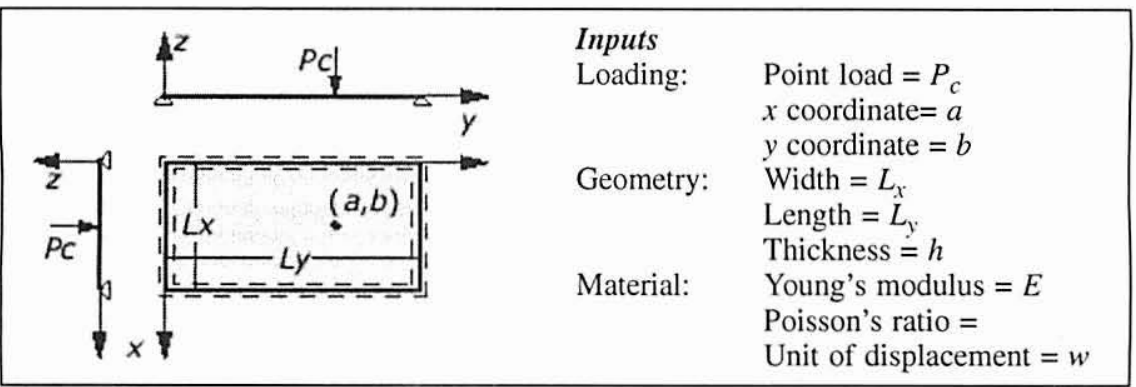

Figure 4

The Model of a Simply Supported Rectangular Plate under a Point Load and Inputs to the Model
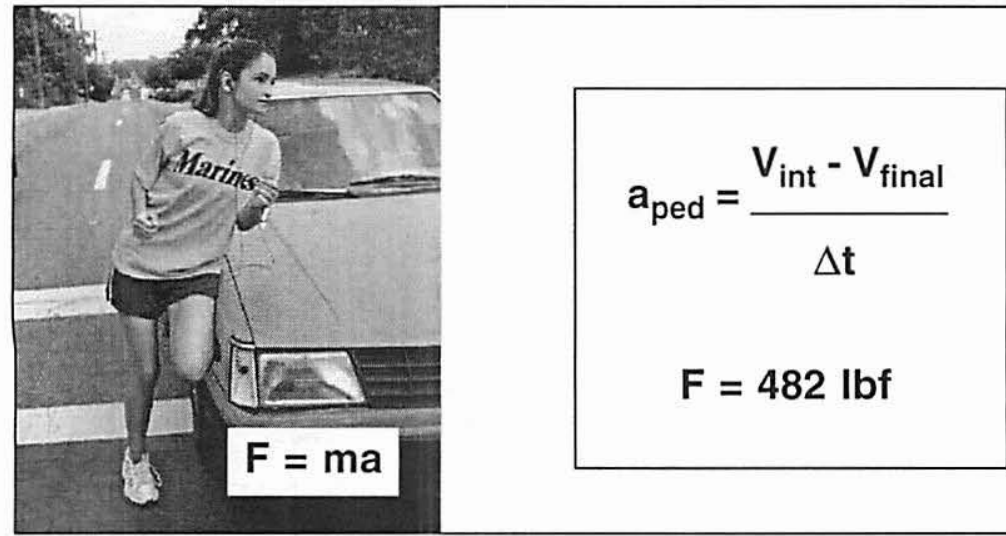

Figure 5

Pedestrian Quantification of Force into the Right Front Quarter Panel 
Conservatively, the minimum force was produced when the pedestrian's lower trunk and bilateral thigh mass impacted the fender (Figure 5). Lastly, the equation in Figure 6 approximated the distortion and indicated that there was likely sufficient force available to cause the indentation in the right front quarter panel. Since the model was only a first order approximation, the conclusion was conservatively stated as the amount of force generated by the pedestrian was consistent with the indentation seen in the defendant's quarter panel.

The analysis and trauma mapping revealed that the pedestrian most likely ran into the side of the sedan at which time the pedestrian's body rotated and fell in a backward fashion with her posterior cranium (back of the head) impacting the right front passenger side A-Pillar of the vehicle (Figure 7). The physical evidence of skull and brain trauma was consistent with A-Pillar impact. The trauma mapping and the physical evidence on the defendant's vehicle are consistent with the pedestrian running into the side of the vehicle.

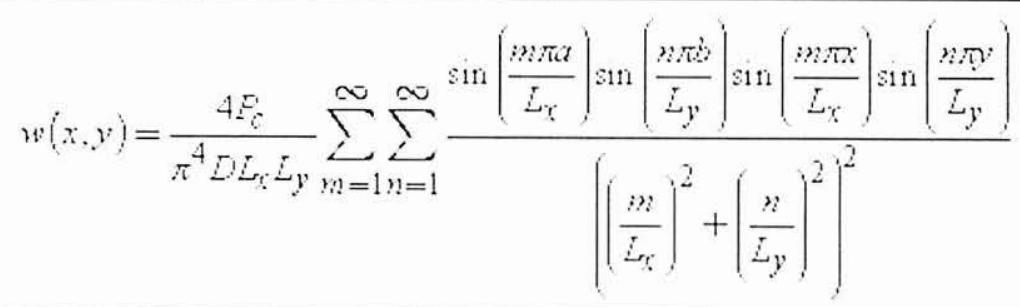

Figure 6

The Equation Utilized to Approximate the Distortion in a Simply Supported Rectangular Plate
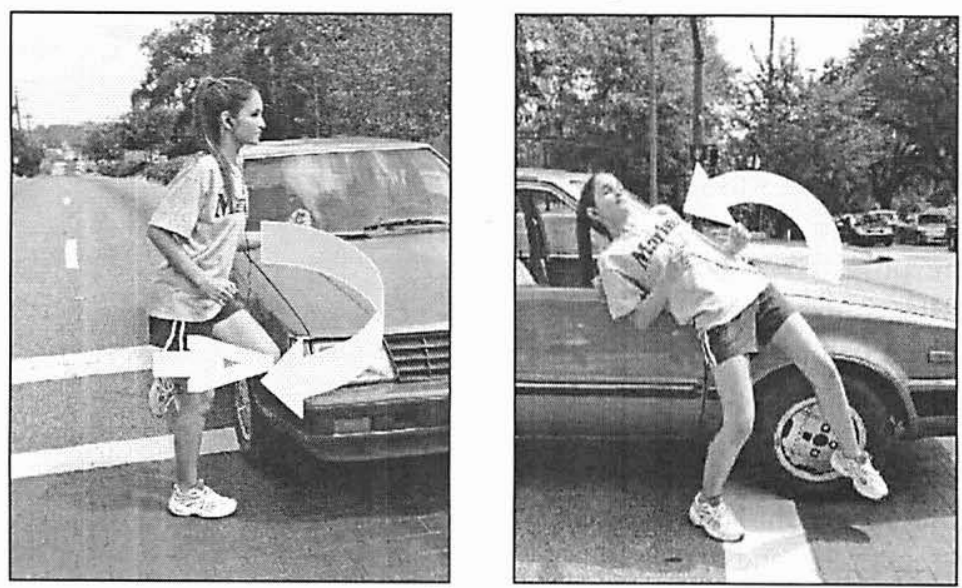

Figure 7

Causes of the Trauma Illustrated with the Model: Back Contacts Antenna, Head Contacts A-Pillar and Elbow/Walkman Contacts Windshield 


\section{Kinematic Study Findings from Both the Accident Reconstruction and BioMedical Engineers}

To determine the line of sight as well as illustrate how the pedestrian's trauma mapped onto the vehicle, a kinematic study proved invaluable as seen in Figures $1 \mathrm{~A}, 5,7,8$ and 9 . The road was closed so that the vehicles could be safely placed and photographed to determine if there were any vehicular or pedestrian blind spots. Procedurally, all the vehicles were put in place on the actual roadway and a model of like height and weight was utilized to represent the pedestrian. This test was conducted during the same calendar week of the actual incident. It was determined that the sun/lighting and foliage effects were non-contributory. The kinematic study revealed that:

- the pedestrian's trauma was consistent with the pedestrian running into the side of the defendant's vehicle and causing the dent in the right front quarter panel;

- the pedestrian traveled for two (2) seconds from the curb to point of impact and the defendant required more than two (2) seconds to swerve or come to a complete stop. Therefore, once the pedestrian left the curb, the defendant could not react in time; and

- the pedestrian was not visible to the defendant at one point five (1.5) seconds (because she was hidden behind the stopped SUV, as shown in Figure $8)$. Also the vehicle was not visible to the pedestrian during the one point five (1.5) seconds prior to

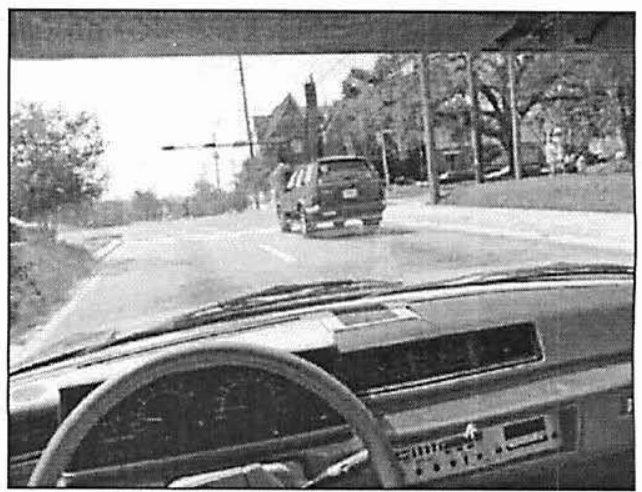

Figure 8

Pedestrian in Defendant's Blind Spot

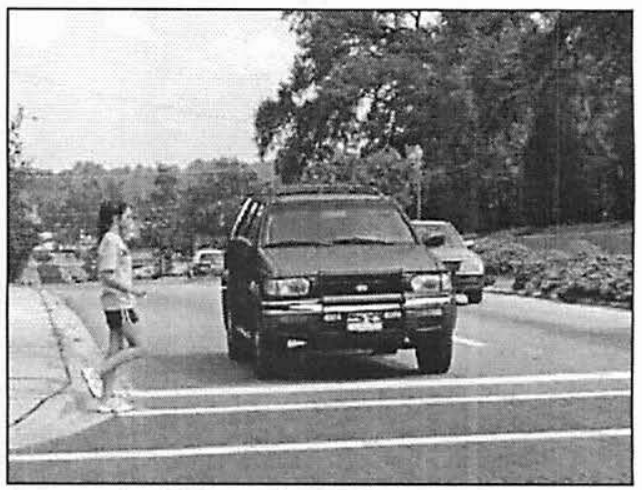

Figure 9

Defendant Vehicle in Pedestrian's Blind Spot impact because she was on the other side of the SUV, as shown in Figure 9. This was consistent with the defendant not knowing what had hit him and the pedestrian running into the side of the defendant's vehicle. 


\section{Conclusions}

From the collective analysis of both the Accident Reconstruction Engineer and the BioMedical Engineer, the following three conclusions were drawn:

1. Blind Spot for both the vehicle and pedestrian occurred at one point five (1.5) seconds prior to impact. The vehicle and the pedestrian were not visible to each other at the last chance to perceive and react;

2. Pedestrian was crossing on a 'Don't Walk" (= Red Hand). The witnesses indicated that both the NB and SB traffic lights were green, and the occupants in the stopped SUV did not see the pedestrian push any buttons; and

\section{Only the Pedestrian Could Have Avoided this Incident}

- The pedestrian was crossing on a "Don't Walk" (= Red Hand).

- The headphones worn by the pedestrian may have masked the auditory cautionary stimulus that a vehicle was approaching.

- Shortly after $(.5 \mathrm{sec})$ the pedestrian left the curb, both the vehicle and pedestrian were in each other's blind spots and then did not have sufficient perception and reaction time to avoid the incident. As a practical matter, this explains why the pedestrian ran into the defendant's vehicle, and the defendant never knew what hit his vehicle.

- The vehicle couldn't have avoided the incident. The last point in time that the defendant had to react and avoid this collision was approximately zero point nine (0.9) seconds before the pedestrian left the curb. This was based on a speed of 25 miles per hour of the defendant's vehicle, a perception-reaction time of one point five (1.5) seconds utilizing maximum braking ( $\mathrm{f}=0.8$ ), and the pedestrians time to the collision from the curb is two (2) seconds. Time to stop = PRT + time to brake, or 1.5 seconds + $(25)(1.467) /(32.2)(0.8)=2.9$ seconds.

The plaintiff's accident reconstruction analysis (without BioMedical Engineering) of this incident resulted in the opinion that the defendant's vehicle hit the pedestrian (plaintiff). Analysis of the physical evidence by both an Accident Reconstruction Engineer and a BioMedical Engineer together indicated that the pedestrian ran into the vehicle.

The authors would like to recognize Kathy Maus and Carolyn Sarginger for the opportunity to have worked on this case and for the permission to use the resulting findings/data in this paper.

Case Result: Following the depositions of both authors, the case was settled at mediation. 


\section{References}

Avallone, E.A., Baumeister, T. (eds.) (1997) Marks Standard Handbook for Mechanical Engineers, 11th ed., McGraw-Hill, Inc. (New York).

Collins, J. (1979), Accident Reconstruction, Charles C. Thomas (Springfield, IL).

Gere, J.M., Timoshenko, S.P. (1984), Mechanics of Materials, 2nd ed., Brooks/Cole (Monterey, CA).

Mase, G.E. (1970), Theory and Problems of Continuum Mechanics, McGrawHill, Inc., (New York).

Pilkey, W.D. (1994), Formulas for Stress, Strain, and Structural Matrices, Wiley (New York).

Van Vlack, L.H. (1985), Elements of Materials Science and Engineering, Addison-Wesley (Reading, MA). 